This item was submitted to Loughborough's Research Repository by the author.

Items in Figshare are protected by copyright, with all rights reserved, unless otherwise indicated.

\title{
Remarks on a strongly nonlinear model for two-layer flows with a top free surface
}

PLEASE CITE THE PUBLISHED VERSION

https://doi.org/10.1111/sapm.12104

PUBLISHER

(c) Wiley

VERSION

AM (Accepted Manuscript)

\section{PUBLISHER STATEMENT}

This work is made available according to the conditions of the Creative Commons Attribution-NonCommercialNoDerivatives 4.0 International (CC BY-NC-ND 4.0) licence. Full details of this licence are available at: https://creativecommons.org/licenses/by-nc-nd/4.0/

\section{LICENCE}

CC BY-NC-ND 4.0

\section{REPOSITORY RECORD}

Barros, Ricardo. 2019. "Remarks on a Strongly Nonlinear Model for Two-layer Flows with a Top Free Surface". figshare. https://hdl.handle.net/2134/33835. 


\title{
Remarks on a strongly nonlinear model for two-layer flows with a top free surface
}

\author{
Ricardo Barros \\ Mathematics Applications Consortium for Science and Industry (MACSI) \\ Department of Mathematics and Statistics \\ University of Limerick \\ Limerick, Ireland
}

\begin{abstract}
We revisit in this manuscript the strongly nonlinear long wave model for large amplitude internal waves in two-layer flows with a free surface proposed by Choi \& Camassa [1] and Barros, Gavrilyuk \& Teshukov [2]. Its solitary-wave solutions were the object of the work by Barros \& Gavrilyuk [3], who proved that such solutions are governed by a Hamiltonian system with two degrees of freedom. A detailed analysis of the critical points of the system is presented here, leading to some new results. It is shown that conjugate states for the long wave model are the same as those predicted by the fully nonlinear Euler equations. Some emphasis will be given to the baroclinic mode, where interfacial waves are known to change polarity according to different values of density and depth ratios. A critical depth ratio separates these two regimes and its analytical expression is derived directly from the model. In addition, we prove that such waves cannot exist throughout the whole range of speeds.
\end{abstract}

\section{Introduction}

Internal waves are a fascinating geophysical phenomenon that has been increasingly appreciated as it is believed to be responsible for a significant fraction of the mixing that must exist to maintain the observed ocean circulation. Weakly nonlinear models, and in particular the (uni-directional) KdV model, have played a crucial role in the early investigations of these waves (see [4] and references therein). However, internal solitary waves often have amplitudes comparable to the thickness of the well-mixed upper layer, which limits considerably the validity of these models [5]. Hence, the need for more elaborate higher-order nonlinear models allowing a more accurate description of internal waves.

The simplest physical system supporting this kind of waves is a two-layer system bounded by two rigid walls. Among the different higher-order nonlinear models that have been proposed to describe the nonlinear waves in this system, we single out the strongly nonlinear model first proposed by Miyata [6], and later by Choi \& Camassa [7] (see [8,9, 10] for related models). The model not only has a rich mathematical structure, but also describes remarkably well the large amplitude solitary wave profiles observed in laboratory experiments and predicted by numerical solutions of the Euler equations [11, 12], while retaining some of the simplicity of weakly nonlinear models.

In oceanic conditions, where the density ratio is close to 1 , the rigid-lid approximation is widely accepted as a good approximation. In laboratory experiments, however, the density ratios can be considerably smaller, and knowing whether or not this approximation is still accurate is a genuine concern. Different authors have proposed models to study the evolution of internal waves in the presence of a top free surface (see $[13,1,14,2,15,16]$ ), but the study of their solutions is rather limited and the free surface effects remain to be more closely examined. 
With recourse to the weakly nonlinear theory, two main effects caused by the presence of a free surface have been detected. One has to do with the polarity of an interfacial wave (in the baroclinic mode) which, as pointed out by Walker [17], is very sensitive to the upper boundary condition (see also $[18,17,19,20])$. The other is the presence of generalized solitary waves, which are simply absent in the rigid-lid case. These have been carefully studied analytically and numerically for the full Euler equations [21, 22], but also in reduced models [23, 20,24], and consist on solitary waves with nondecaying oscillatory tails. The particular case when ripples in the far field in addition to the solitary pulse vanish is considered to be rare and, perhaps for this reason, the study of pure solitary-wave solutions have been mostly restricted to the barotropic mode $[25,26]$. Nonetheless, evidence of pure solitary-wave solutions in the baroclinic mode have been provided by Barros \& Gavrilyuk [3] and Percival, Cotter \& Holm [15].

This present work was motivated by a number of questions that remained open in [3]. After introducing in $\S 2$ the mathematical model and presenting some basic considerations, we give in $\S 3$ a detailed analysis of the critical points of the dynamical system governing its solitary-wave solutions, which previously had only been investigated numerically. The critical points are examined regarding their location, number, and nature. A particular emphasis is given to the baroclinic mode, where a critical depth ratio determining the polarity of the interfacial wave is identified in $\S 4$ and related to a structural change of the potential for the Hamiltonian system. For the first time, the analytical expression of this critical value is derived explicitly from the model and it is shown to be in agreement with what has been known for the weakly nonlinear theory. Moreover, by using the results on the collision of critical points it is revealed that solitary waves in this mode cannot exist for the whole range of speeds.

Finally, $\S 5$ is devoted to the conjugate states predicted by the model and how well these compare with results obtained by Dias \& Il'ichev [20] for the full Euler equations. Notice that if front waves connecting two conjugate states exist for Euler equations, they should be particularly well described by our model, since for being a long wave model (with no restriction on the magnitude of wave amplitude), the broader the waves are, the better is the expected agreement between the two. This idea is corroborated here and it is established that the conjugate states are exactly the same in both models.

\section{A strongly nonlinear model for internal waves and some basic considerations}

The one-dimensional version of the strongly nonlinear long wave model governing the evolution of gravity waves at the interface and free surface of two immiscible inviscid fluids, with constant densities $\rho_{i}$, is written in terms of layer thicknesses $h_{i}$ and depth-averaged velocities $\bar{u}_{i}(i=1$ and 2 represent the lower and upper layers, respectively) as follows (see $[1,2])$ :

$$
\begin{gathered}
h_{1 t}+\left(h_{1} \bar{u}_{1}\right)_{x}=0, \quad h_{1}=H_{1}+\zeta_{1}(x, t) \\
h_{2 t}+\left(h_{2} \bar{u}_{2}\right)_{x}=0, \quad h_{2}=H_{2}+\zeta_{2}(x, t)-\zeta_{1}(x, t), \\
\bar{u}_{1 t}+\bar{u}_{1} \bar{u}_{1 x}+g\left(h_{1}+\rho h_{2}\right)_{x}+\rho\left(-\frac{1}{2} G_{2} h_{2}^{2}+\left(D_{2}^{2} h_{1}\right) h_{2}\right)_{x}=\frac{1}{3 h_{1}}\left(h_{1}^{3} G_{1}\right)_{x}, \\
\bar{u}_{2 t}+\bar{u}_{2} \bar{u}_{2 x}+g\left(h_{1}+h_{2}\right)_{x}=\frac{1}{3 h_{2}}\left(h_{3}^{2} G_{2}\right)_{x}-\frac{1}{2 h_{2}}\left(h_{2}^{2} D_{2}^{2} h_{1}\right)_{x}+\left(\frac{1}{2} h_{2} G_{2}-D_{2}^{2} h_{1}\right) h_{1 x} .
\end{gathered}
$$

Here, $g$ is the gravitational acceleration, $\rho=\rho_{2} / \rho_{1}(<1)$ is the depth ratio, $\zeta_{i}(x, t)$ are the displacements of the interface $(i=1)$ and top free surface $(i=2)$, and the subscripts $x$ and $t$ represent partial differentiation with respect to space and time, respectively. The model is a two-layer generalization of the $\mathrm{Su}$-Gardner equations [28] (also referred to as the Green-Naghdi equations [29] in the literature), and can be thought of as the two-layer shallow water equations modified to include nonlinear dispersive effects, here accounted for by

$$
G_{i}=\bar{u}_{i x t}+\bar{u}_{i} \bar{u}_{i x x}-\bar{u}_{i x}{ }^{2},
$$


and $D_{2}^{2} h_{1}$, denoting the second-order material derivative of $h_{1}$ with respect to $\bar{u}_{2}$, with $D_{2}=\partial_{t}+\bar{u}_{2} \partial_{x}$.

We remark that the model can be obtained from the original Euler equations for weakly rotational flows under the long-wave approximation. For this reason, while it can accurately describe long interfacial and surface wave phenomena, it fails to describe short wave disturbances such as the internal wave signatures that can be observed in the ocean (see e.g. [30]).

By linearizing the system (1) about $\bar{u}_{i}=0$ and $h_{i}=H_{i}$, and examining the dispersion relation, it can be seen that, provided the stratification is stable $(\rho<1)$, the wave speed $c$ is always real and two branches of solutions are found for right propagating waves. These two branches characterize two different modes, the so-called baroclinic and barotropic modes, according to the magnitude of the wave speed. In the long wave limit, one obtains in non-dimensional variables

$$
\left(F^{2}-1\right)\left(F^{2}-H\right)-\rho H=0,
$$

by setting the Froude number $F$ and depth ratio $H$ as

$$
F^{2}=c^{2} / g H_{1}, \quad H=H_{2} / H_{1} .
$$

The roots of Eq. (2) yield the well-known linear long wave speeds

$$
F_{ \pm}^{2}=\frac{H+1 \pm \sqrt{(H-1)^{2}+4 \rho H}}{2},
$$

whose larger value satisfies $F_{+}^{2}>1$ and $F_{+}^{2}>H$, while $F_{-}^{2}<1-\rho$ and $F_{-}^{2}<H(1-\rho)$ (see [20]).

A remarkable feature of the system is that it has a Hamiltonian structure, which is also preserved for its solitary-wave solutions. To seek these particular solutions of (1), we may use the conservative form for the momentum equations, and plug in the equations the ansatz $h_{i}=h_{i}(x-c t), \bar{u}_{i}=\bar{u}_{i}(x-c t)$, by imposing the boundary conditions $h_{i} \rightarrow H_{i}, \bar{u}_{i} \rightarrow 0$ as $\xi=x-c t$ goes to infinity. By doing so, one obtains the dynamical system [3]:

$$
\begin{gathered}
F^{2}\left[\frac{1}{6}\left(\frac{h_{1}^{\prime}}{h_{1}}\right)^{2}+\frac{1}{3}\left(\frac{h_{1}^{\prime}}{h_{1}}\right)^{\prime}+\rho H\left(\frac{h_{1}^{\prime}}{h_{2}}\right)^{\prime}+\frac{1}{2} \rho H^{2}\left(\frac{h_{2}^{\prime}}{h_{2}}\right)^{\prime}\right]=-\frac{\partial V}{\partial h_{1}}, \\
\rho H F^{2}\left[\frac{1}{2}\left(\frac{h_{1}^{\prime}}{h_{2}}\right)^{2}+\frac{H}{2}\left(\frac{h_{1}^{\prime}}{h_{2}}\right)\left(\frac{h_{2}^{\prime}}{h_{2}}\right)+\frac{H^{2}}{6}\left(\frac{h_{2}^{\prime}}{h_{2}}\right)^{2}+\frac{H}{2}\left(\frac{h_{1}^{\prime}}{h_{2}}\right)^{\prime}+\frac{H^{2}}{3}\left(\frac{h_{2}^{\prime}}{h_{2}}\right)^{\prime}\right]=-\frac{\partial V}{\partial h_{2}},
\end{gathered}
$$

written in non-dimensional form, where prime denotes differentiation with respect to $\xi^{*}=\xi / H_{1}$, $V\left(h_{1}, h_{2}\right)$ is the potential defined by

$V\left(h_{1}, h_{2}\right)=-\frac{1}{2} F^{2} \frac{\left(h_{1}-1\right)^{2}}{h_{1}}+\frac{1}{2}\left(h_{1}-1\right)^{2}+\rho H\left[-\frac{1}{2} F^{2} \frac{\left(h_{2}-1\right)^{2}}{h_{2}}+\frac{1}{2} H\left(h_{2}-1\right)^{2}\right]+\rho H\left(h_{1}-1\right)\left(h_{2}-1\right)$,

and the depth ratio $H$ and Froude number $F$ are given as in (3). It is worth to point out that this particular potential was chosen in order to verify simultaneously

$$
V(1,1)=0, \quad \nabla V(1,1)=0,
$$

and each thickness of the layers has been scaled (in a non-standard way) as $h_{i}=H_{i} h_{i}^{*}$ to achieve this purpose. We also note that all variables in (5) should be adorned with asterisks, but these have been dropped for convenience.

In the following sections, we present a detailed study of the critical points of (5) and reveal some interesting consequences of the analysis. 


\section{Critical point analysis}

Before we proceed, we would like to give a flavor of what can be learned about the solutions of our dynamical system, by relying solely on its Hamiltonian structure and critical point analysis. Consider the particular case when $\rho=0$ (single-layer flow). The system (5) reduces to

$$
\frac{1}{6} F^{2}\left[h_{1}^{-1 / 2} h_{1}^{\prime 2}\right]^{\prime}=-\left[\frac{1}{2} F^{2} \frac{1-h_{1}^{2}}{h_{1}^{2}}+\left(h_{1}-1\right)\right] h_{1}^{\prime}
$$

and can be integrated once to yield

$$
\frac{1}{2}\left(h_{1}^{\prime}\right)^{2}=-\frac{3}{2 F^{2}}\left(h_{1}-1\right)^{2}\left(h_{1}-F^{2}\right) \equiv-\widetilde{V}\left(h_{1}\right),
$$

which can be interpreted as the motion of a particle with mass one and zero total energy in a field whose potential is given by $\widetilde{V}$. This is very useful since it can be established at once that solitary-wave solutions exist if and only if $F^{2}>1$, which amounts to impose that the critical point $h_{1}=1$ is a local maximum of $\widetilde{V}$. Given this condition, it can be checked that the other critical point $h_{1}=\left(1+2 F^{2}\right) / 3$ $(>1)$ is a local minimum of $\widetilde{V}$. Homoclinic trajectories are obtained whenever a particle leaves the equilibrium $h_{1}=1$ towards the right, where the minimum is located. Thus, solitary waves exist for any supercritical flow and are waves of elevation with amplitude $F^{2}-1$.

When $\rho \neq 0$, the dynamical system (5) is equivalent to a Hamiltonian system with two degrees of freedom. The richness and complexity of the dynamics of the system have been displayed in [3] through numerous numerical solutions, where features such as broadening of the waves and existence of multi-humped solutions were exhibited. A first integral of (5) is given by the Hamiltonian $\mathbb{H}=$ $T\left(h_{1}, h_{2}, h_{1}^{\prime}, h_{2}^{\prime}\right)+V\left(h_{1}, h_{2}\right)$, where $V$ is the potential in (6) and $T$ is the kinetic energy defined by

$$
T=\frac{1}{2} F^{2}\left[\begin{array}{ll}
h_{1}^{\prime} & h_{2}^{\prime}
\end{array}\right]\left[\begin{array}{cc}
\frac{1}{3 h_{1}}+\rho H \frac{1}{h_{2}} & \frac{1}{2} \rho H^{2} \frac{1}{h_{2}} \\
\frac{1}{2} \rho H^{2} \frac{1}{h_{2}} & \frac{1}{3} \rho H^{3} \frac{1}{h_{2}}
\end{array}\right]\left[\begin{array}{c}
h_{1}^{\prime} \\
h_{2}^{\prime}
\end{array}\right] .
$$

Finding another first integral for the system is extremely unlikely, and so are the chances of providing explicit analytical solutions, or even a relationship between the wave speed and wave amplitudes. However, similarly to the simpler case when $\rho=0$, some properties of solutions can still be inferred from a critical point analysis, which is what we propose to do here.

The critical points of (5) are precisely the critical points of $V$. These are found as solutions of $\nabla V\left(h_{1}, h_{2}\right)=(0,0)$, and can be classified by examining the Hessian matrix of $V$, here denoted by $\mathcal{H}$ :

$$
\mathcal{H}=\left[\begin{array}{cc}
-F^{2} / h_{1}^{3}+1 & \rho H \\
\rho H & \rho H\left(-F^{2} / h_{2}^{3}+H\right)
\end{array}\right] .
$$

From (7), $\nabla V(1,1)=0$, point at which $\operatorname{det} \mathcal{H}$ is just a multiple of the left-hand side of (2). As a result, $(1,1)$ is a non-degenerate critical point for the range of speeds $\mathbb{R}^{+} \backslash\left\{F_{ \pm}\right\}$. It becomes a saddle point of $V$ when $\operatorname{det} \mathcal{H}(1,1)<0$, i.e., when $F_{-}<F<F_{+}$, and a local extremum otherwise. By observing that $F_{-}^{2}<1<F_{+}^{2}$, it immediately follows that $(1,1)$ is a local minimum within $] 0, F_{-}[$, and a local maximum within $] F_{+}, \infty[$.

Characterizing the remaining critical points is slightly more tricky. This task will be accomplished in a few steps. First we find their geometrical location, then examine when they collide, and finally determine their number and nature.

\subsection{Geometrical locus of critical points}

Since $h_{1}, h_{2}$ are physical variables denoting the thickness of each layer, both are assumed to be strictly positive. For a given Froude number, the set of critical points can then be determined as the 
intersection (in the first quadrant) of two cubic curves:

$$
\begin{gathered}
\mathcal{C}_{1}\left(h_{1}, h_{2}\right) \equiv \frac{1}{2} F^{2}\left(1-h_{1}^{2}\right)+h_{1}^{2}\left(h_{1}-1\right)+\rho H h_{1}^{2}\left(h_{2}-1\right)=0, \\
\mathcal{C}_{2}\left(h_{1}, h_{2}\right) \equiv \frac{1}{2} F^{2}\left(1-h_{2}^{2}\right)+h_{2}^{2}\left(h_{1}-1\right)+H h_{2}^{2}\left(h_{2}-1\right)=0 .
\end{gathered}
$$

From these, the Froude number can be eliminated to give the following relationship between $h_{1}$ and $h_{2}$ :

$$
P\left(h_{1}, h_{2}\right) \equiv h_{1}^{3}+\left[-1+H\left(h_{2}-1\right) h_{2}^{2}+\rho H\left(h_{2}-1\right)\left(1-h_{2}^{2}\right)\right] h_{1}^{2}-h_{2}^{2} h_{1}+h_{2}^{2}\left[1-H\left(h_{2}-1\right)\right]=0 .
$$

In the first quadrant, Eqs. (9)-(10) and (10)-(11) have the exact same solutions. In particular, any critical point lies on the geometrical locus defined by the curve $P=0$ in (11). As we will see, the converse implication also holds and a point with coordinates $\left(h_{1}, h_{2}\right) \in \mathbb{R}^{+} \times \mathbb{R}^{+}$is a critical point of $V$ (for some wave speed) if and only if $P\left(h_{1}, h_{2}\right)=0$.

Clearly, any point of the first quadrant verifying $P=0$ can be used to find a value of $F^{2}$ such that (10) holds, by setting

$$
F^{2}=-\frac{2 h_{2}^{2}}{1-h_{2}^{2}}\left[h_{1}-1+H\left(h_{2}-1\right)\right] .
$$

The only issue here is identifying whether or not such value is admissible; in other words, if the right-hand side of (12) is or not positive.

Let $l$ be the line defined by

$$
l \equiv h_{1}-1+H\left(h_{2}-1\right)=0 .
$$

To prove our claim it suffices to show that the curve $P=0$ is above (below) $l$ for $h_{2}>1\left(0<h_{2}<1\right)$. We start by observing that locally at the point $(1,1)$ the curve $P=0$ is described by

$$
\left(h_{1}-1\right)^{2}+(H-1)\left(h_{1}-1\right)\left(h_{2}-1\right)-\rho H\left(h_{2}-1\right)^{2}=0,
$$

which splits up into two lines

$$
\left(h_{1}-1\right)-v_{0}^{ \pm}\left(h_{2}-1\right)=0
$$

with $v_{0}^{ \pm}=F_{ \pm}^{2}-H$, which are the tangents to the curve at the crunode $(1,1)$. Therefore, at least locally at the point $(1,1)$, the curve is above (below) $l$ for $h_{2}>1\left(0<h_{2}<1\right)$. The result can then be extended to whole quadrant by noticing that, in this region, $l$ intersects $P=0$ nowhere else besides the point $(1,1)$.

\subsection{Collision of critical points}

Given fixed values of $\rho$ and $H$, the potential $V$ defined in (6) is in fact a one-parameter family of functions. As the Froude number varies, the surface of the potential $V$ is smoothly deformed and the number of critical points remains invariant unless a collision of critical points takes place. Examining when collisions occur is thus a crucial step in the critical point analysis.

Critical points can be determined in two different ways. One way consists in prescribing any point of $P=0$ (within the first quadrant) and find the corresponding wave speed from (12). Another way is to fix the value of speed and find the points on the curve $P=0$ that are also solutions of $\mathcal{C}_{2}=0$. To do so, from (10), extract the value of $h_{1}$ as a function of $h_{2}$, and substitute this into (9). As a result, the candidates for critical points have ordinates $h_{2}$ that are solutions of

$$
\left(h_{2}-1\right) P_{8}\left(h_{2}\right)=0,
$$

where $P_{8}$ is an eighth-degree polynomial on $h_{2}$, i.e., $P_{8}=\sum_{k=0}^{8} b_{k} h_{2}^{8-k}$, with coefficients:

$$
b_{0}=8 H^{3}(1-\rho), \quad b_{1}=-8 H^{2}(1-\rho)\left(2+2 H+F^{2}\right), \quad b_{2}=2 H(1-\rho)\left(2+2 H+F^{2}\right)^{2}
$$




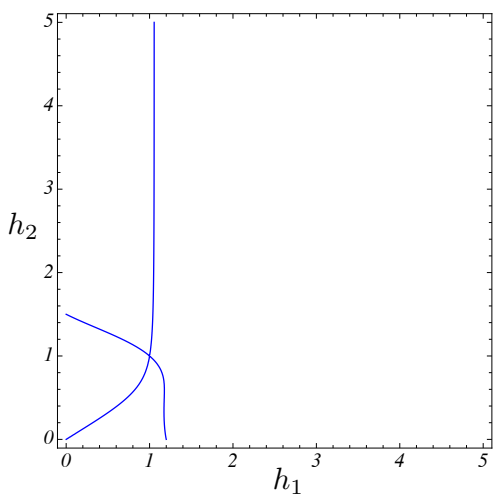

(a)

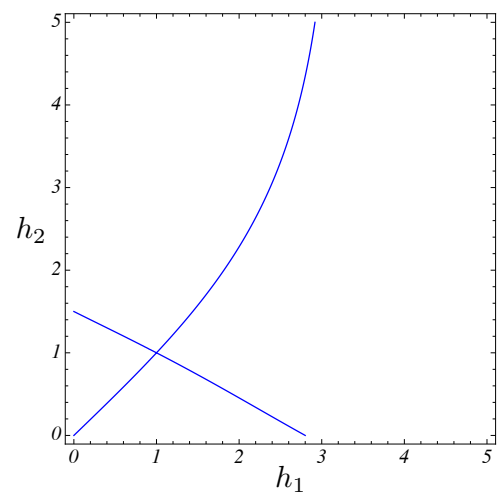

(b)

Figure 1: Plots on the $\left(h_{1}, h_{2}\right)$-plane of the curve $P=0$ defined by Eq. (11), consisting of the geometrical locus of all critical points of the potential $V$. (a) $\rho=0.1, H=2$, (b) $\rho=0.9, H=2$.

$$
\begin{gathered}
b_{3}=-4\left(1-3 H^{2}+2 \rho H^{2}\right) F^{2}, \quad b_{4}=4\left[-1-4 H-3 H^{2}+2 \rho H(1+H)+H(\rho-2) F^{2}\right] F^{2}, \\
b_{5}=\left(4+F^{2}\right) F^{4}, \quad b_{6}=\left(4+6 H-2 \rho H+F^{2}\right) F^{4}, \quad b_{7}=-F^{6}, \quad b_{8}=-F^{6} .
\end{gathered}
$$

Remark that a collision of critical points is produced whenever (14) has multiple roots. In particular, by examining the case when $h_{2}=1$ is a double root of (14), or equivalently $P_{8}\left(h_{2}=1\right)=0$, leads to the condition

$$
8\left[F^{4}-(1+H) F^{2}+(1-\rho) H\right]=0,
$$

which as we know is met precisely at the linear long wave speeds $F=F_{ \pm}$. On the other hand, if $h_{2}=1$ is a triple root of $(14)$, then not only $P_{8}\left(h_{2}=1\right)=0$, but also

$$
P_{8}^{\prime}\left(h_{2}=1\right)=4\left\{F^{6}+F^{4}(5-2 H)+\left[-9+H^{2}-4 H(1+\rho)\right] F^{2}+4(\rho-1) H(H-3)\right\}=0,
$$

for a certain Froude number. This implies that the resultant between $P_{8}\left(h_{2}=1\right)$ and $P_{8}^{\prime}\left(h_{2}=1\right)$ with respect to $F^{2}$ is zero [32], i.e.,

$$
73728 H(1-\rho)\left[H^{3}-\left(3-3 \rho-\rho^{2}\right) H^{2}+(3-4 \rho) H-1\right]=0 .
$$

The cubic expression in brackets will appear later in the text (see $\S 4$ ) and it will be shown to govern the polarity of an interfacial wave in the baroclinic mode.

We now identify other possible collisions. Let $h_{2}$ be a double root of $P_{8}\left(h_{2}\right)$. Then, the discriminant of $P_{8}$ with respect to $h_{2}$ must vanish. This yields a polynomial equation $P_{12}\left(F^{2}\right)=0$ of degree 12 for $F^{2}$ that implicitly defines the speed values at which collisions take place. The expression of $P_{12}$ is unfortunately too cumbersome to be presented here. However, the reader should be able to reproduce the results displayed in figures 2, 3 by using any symbolic program like MathematicA.

Our extensive numerical tests show that regardless of the values of $\rho$ and $H$, a single root $F_{c}$ of $P_{12}$ can be found below the linear long wave speed $F_{+}$. Namely, $F_{c}$ is within the range $] F_{-}, F_{+}[$, as shown in figure 2. More surprisingly, other collisions can also occur in the barotropic mode, provided $H$ is small enough ${ }^{1}$ (see figure 3 ).

Some care is needed in interpreting these results. Notice that in $\mathbb{C}$ two polynomials have a common root if and only if their resultant vanishes [32]. However, the field of real numbers is not algebraically

\footnotetext{
${ }^{1}$ The threshold for $H$ below which these extra collisions arise has been found to decrease with $\rho$ and, in particular when $\rho>0.9$, such value of $H$ is less than 0.13 .
} 


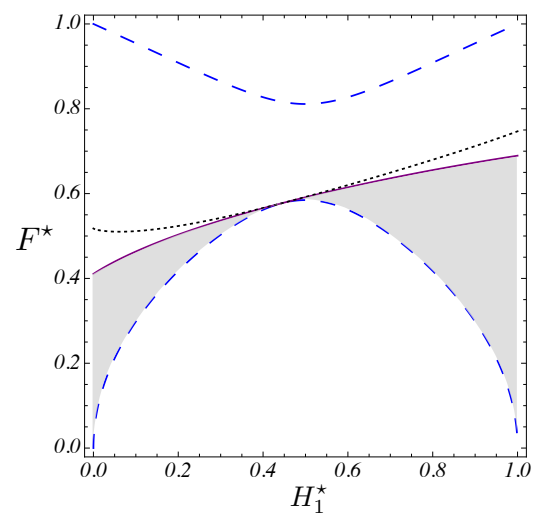

(a)

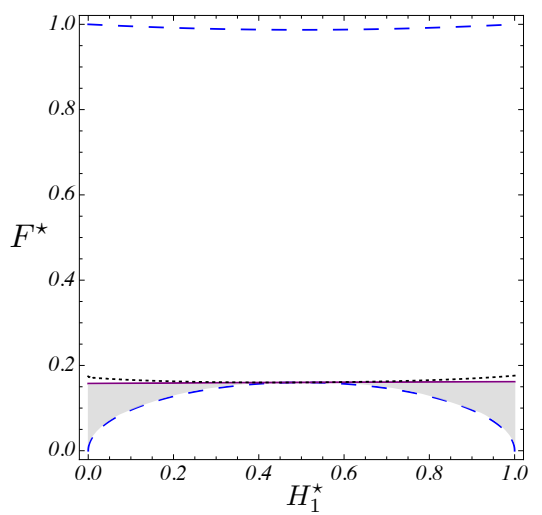

(b)

Figure 2: Wave speeds at which critical points collide, and those at which front-like solutions may exist. The dashed lines represent the linear long wave speeds $F=F_{ \pm}$, at which there is a collision at the point $(1,1)$. Other possible collisions (occurring at the speed $F=F_{c}$ ) are represented by the dotted line. The full line shows the speed $F=F_{\text {front }}$ at which Eq. (24) is non-trivially satisfied and conjugate states exist (see $\S 5$ ). The light shaded region corresponds to the realm of existence of solitary waves conjectured by Dias \& Il'ichev [20] for the Euler equations. The diagram is presented on the $\left(H_{1}^{\star}, F^{\star}\right)$-plane with $H_{1}^{\star}=1 /(H+1)$ and $\left(F^{\star}\right)^{2}=F^{2} /(H+1)$. (a) $\rho=0.1,(b) \rho=0.9$.

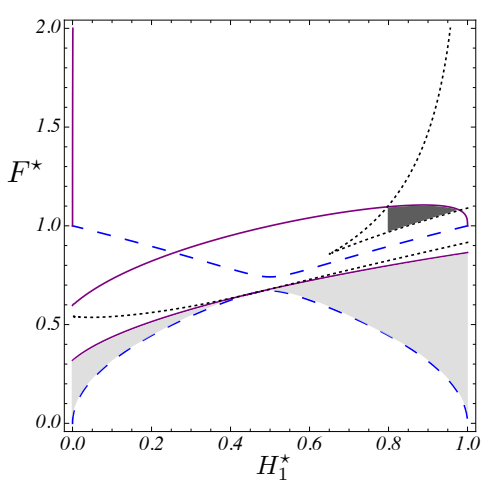

(a)

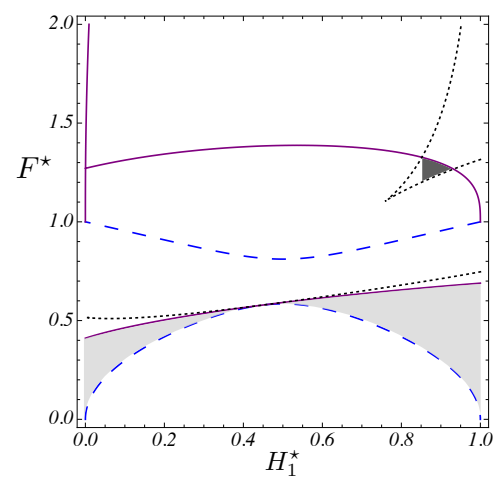

(b)

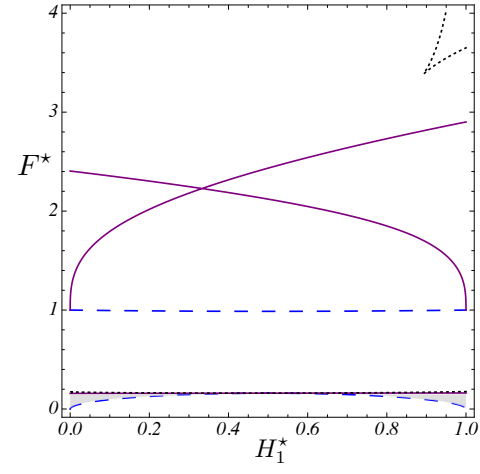

$(c)$

Figure 3: Wave speeds at which critical points collide, and those at which front-like solutions may exist. The dashed lines represent the linear long wave speeds $F=F_{ \pm}$, at which there is a collision at the point $(1,1)$. Other possible collisions are represented by dotted lines. The full lines show the speeds at which Eq. (24) is non-trivially satisfied and conjugate states may exist (see § 5). The light shaded region corresponds to the realm of existence of solitary waves conjectured by Dias \& Il'ichev [20] for the Euler equations. The dark shaded region corresponds to the case where conjugate states in the barotropic mode cannot a priori be ruled out. The diagram is presented on the $\left(H_{1}^{\star}, F^{\star}\right)$-plane with $H_{1}^{\star}=1 /(H+1)$ and $\left(F^{\star}\right)^{2}=F^{2} /(H+1)$. (a) $\rho=0.01,(b) \rho=0.1,(c) \rho=0.9$. Figure 2 is just a blow-up diagram of panels $(b)$ and $(c)$. 
closed. Therefore, even if the resultant vanishes, it could lead to non-admissible solutions, as the values of $h_{1}$ or $h_{2}$ may become negative, or even complex. Nonetheless, this possibility does not apply here and, in fact, each one of these collisions leads to a change on the number of critical points, as discussed further down in the text.

\subsection{Number and nature of critical points}

\subsubsection{Number}

For a given Froude number, the critical points are found as the intersection (in the first quadrant) between two cubic curves. Bézout's Theorem states that two curves with degree $n_{1}, n_{2}$ intersect in $n_{1} n_{2}$ points, provided that each intersection is counted a suitable number of times [33]. This result is important because it shows that the set of critical points is finite and composed by isolated points. Here, however, we expect to have less than nine points, since for physical reasons we confine our study to real geometry and the first quadrant of the plane. We shall prove that the number of critical points can only be two or four.

We start by considering the limit when $F \rightarrow 0^{+}$. In this limit, both $\mathcal{C}_{1}, \mathcal{C}_{2}$ in (9)-(10) become degenerate and their intersection can be easily determined as the set (see figure 5 )

$$
\left.\mathcal{C}_{1} \cap \mathcal{C}_{2}\right|_{F \rightarrow 0^{+}}=\{(0,0),(1,1),(0,1+1 / H),(1+\rho H, 0)\} .
$$

As the wave speed increases, the number of critical points is preserved unless a collision takes place.

A collision can occur at $(1,1)$, with no effect on the number of critical points ${ }^{2}$. Since $(1,1) \in \mathcal{C}_{1} \cap \mathcal{C}_{2}$ regardless of the parameters, it makes sense to determine the tangents to $\mathcal{C}_{1}, \mathcal{C}_{2}$ at this point, given respectively by

$$
\begin{gathered}
\left(h_{1}-1\right)\left(1-F^{2}\right)+\left(h_{2}-1\right) \rho H=0, \\
h_{1}-1+\left(h_{2}-1\right)\left(H-F^{2}\right)=0 .
\end{gathered}
$$

It is straightforward to verify that these lines coincide if and only if (2) holds. A collision at (1,1) can then be interpreted as the condition for $\mathcal{C}_{1}, \mathcal{C}_{2}$ to be tangent at this point.

Furthermore, it can be proved that at $F=F_{+}$the line (16) divides the first quadrant in two separate connected components, each containing only one of the curves $\mathcal{C}_{1}, \mathcal{C}_{2}$ (see figure 4 ). This implies that $\sharp \mathcal{C}_{1} \cap \mathcal{C}_{2}=2$ at the linear long wave speed $F_{+}$, with $(1,1)$ counted twice. Since, initially at $F \approx 0, \sharp \mathcal{C}_{1} \cap \mathcal{C}_{2}=4$, somewhere between 0 and $F^{+}$there has to be a collision responsible for this change. As there is a single eligible candidate, we conclude that the collision curve $F=F_{c}$ as shown in figure 2 is not spurious and leads to a decrease on the number of critical points of $V$.

The next step in our study greatly benefits from the fact of one being able to parametrize different branches of the curve $P=0$. Following Miura [31], it is shown in Appendix A that the curve $P=0$ is parameterized in the first quadrant by $h_{1}=\lambda_{k}\left(h_{2}\right)$, with $k=2,3$ (see figure 5). Let $\Gamma_{k}\left(h_{2}\right)$ the function defined by

$$
\Gamma_{k}\left(h_{2}\right) \equiv \frac{2 h_{2}^{2}}{1-h_{2}^{2}}\left[1-\lambda_{k}\left(h_{2}\right)-H\left(h_{2}-1\right)\right], \quad k=2,3 .
$$

It is clear from (12) that these functions specify the range of speeds attained along each solution branch of $P=0$. Moreover, $\Gamma_{k}\left(h_{2}\right)=2 h_{2}^{2} \Lambda_{k}\left(h_{2}\right) /\left(1-h_{2}^{2}\right)$, where $\left|\Lambda_{k}\left(h_{2}\right)\right|$ is the distance between each parametrized branch of the curve and the line $l$ of equation (13). Clearly, $\Lambda_{k}\left(h_{2}\right)$ is continuous, but loses regularity at $h_{2}=1$, at which is not differentiable. As a result, $\Gamma_{k}\left(h_{2}\right)$ has different lateral limits at $h_{2}=1$ :

$$
\lim _{h_{2} \rightarrow 1^{ \pm}} \Gamma_{2}\left(h_{2}\right)=F_{\mp}^{2}, \quad \lim _{h_{2} \rightarrow 1^{ \pm}} \Gamma_{3}\left(h_{2}\right)=F_{ \pm}^{2} .
$$

\footnotetext{
${ }^{2}$ Because $(1,1)$ is a non-degenerate critical point for all range of speeds $\mathbb{R}^{+} \backslash\left\{F_{ \pm}\right\}$, the number of critical points is preserved upon a collision at $(1,1)$. The only exception to this is when we have a triple collision (see $\S 3.2)$.
} 


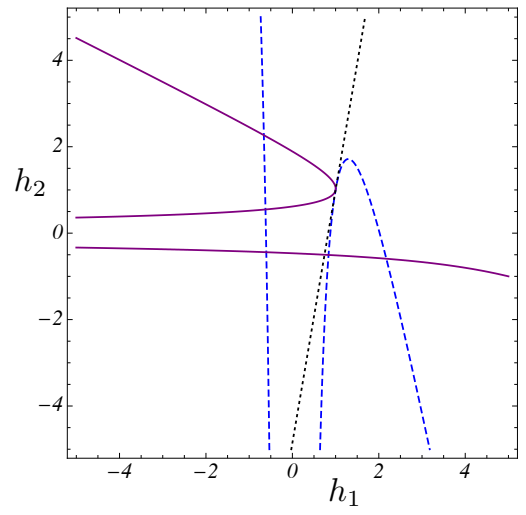

(a)

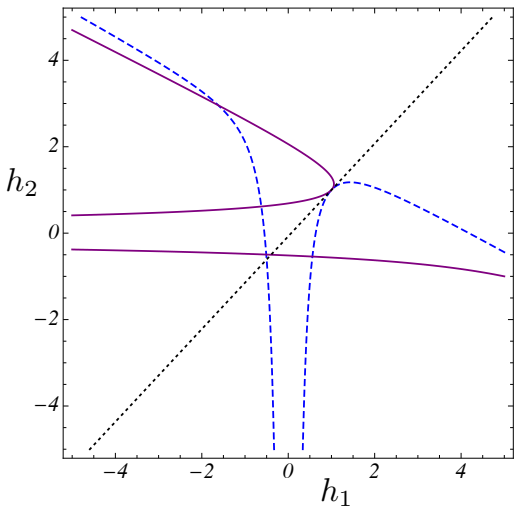

(b)

Figure 4: Tangency at $(1,1)$ between curves $\mathcal{C}_{1}$ (dashed line) and $\mathcal{C}_{2}$ (full line) defined in (9)-(10) with $F=F^{+}$. The tangent line in (16) is represented by a dotted line. $(a) \rho=0.1, H=2,(b) \rho=0.9$, $H=2$.

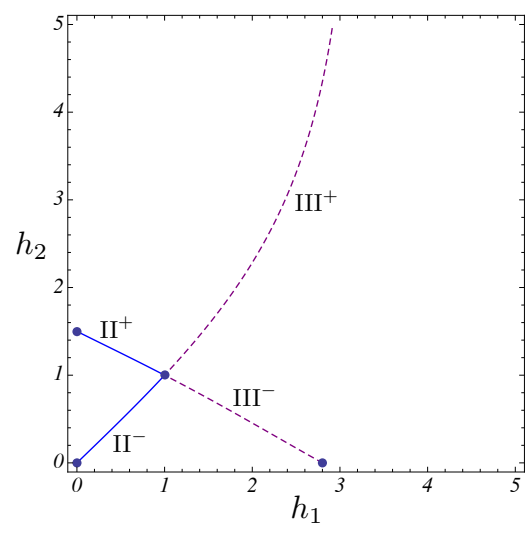

(a)

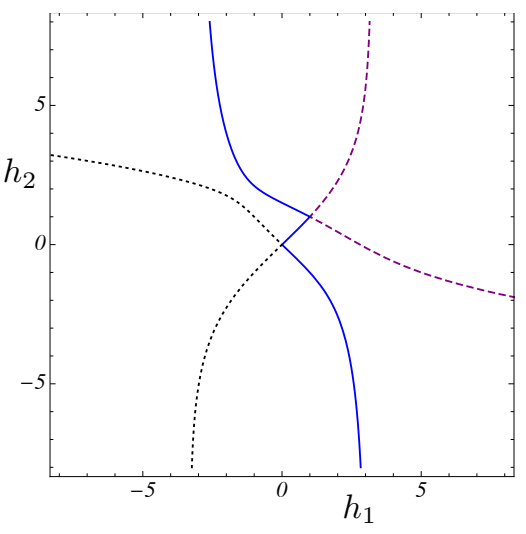

(b)

Figure 5: Parametric plots on the $\left(h_{1}, h_{2}\right)$-plane of the curve $P=0$ with $\rho=0.9, H=2$. In $(a)$, the two solution branches II, III are parametrized by $h_{1}=\lambda_{2}\left(h_{2}\right)$ (full line) and $h_{1}=\lambda_{3}\left(h_{2}\right)$ (dashed line). The four points in the diagram are the solutions of (9)-(10) under the limit $F \rightarrow 0^{+}$in $(15)$. In $(b)$, the curve $P=0$ is plotted on the whole plane, revealing a solution branch parametrized by $h_{1}=\lambda_{1}\left(h_{2}\right)$ (dotted line) that takes only negative values, as proved in Appendix A. The way in which the curve tends to infinity is prescribed by two asymptotes with equations $h_{1}= \pm 1 / \sqrt{1-\rho}$, and an infinite branch. 
Here and hereafter, the cases when $h_{2}>1$ or $h_{2}<1$ will be treated separately for each parameterization $h_{1}=\lambda_{k}\left(h_{2}\right), k=2,3$. Four solutions branches will then be considered (instead of two) and will be denoted by $\mathrm{II}^{ \pm}(k=2)$, or $\mathrm{III}^{ \pm}(k=3)$, where plus and minus signs are chosen for $h_{2}>1$ and $h_{2}<1$, respectively (see figure 5 ).

Observe that if $\Gamma_{k}\left(h_{2}\right)$ is monotonic, then for each value of $F$ such that $F^{2}$ is within the range of the mapping $\Gamma_{k}\left(h_{2}\right)$, there exists one single critical point crossing the whole branch. By contrary, if there is lack of monotonicity, each local extremum of $\Gamma_{k}\left(h_{2}\right)$ will correspond to a collision of critical points.

It follows from (15) and (18) that: $] 0, F_{-}^{2}\left[\right.$ is included in the range of the mappings $\Gamma_{2}$ in $] 1,1+1 / H$, and $\Gamma_{3}$ in $] 0,1[;] 0, F_{+}^{2}$ [ is included in the range of $\Gamma_{2}$ in $] 0,1\left[\right.$. Since there are up to the speed $F=F_{-}$ precisely 4 critical points, then $(1,1)$ is one of them, together with three other points, each one located at a different branch: $\mathrm{II}^{-}, \mathrm{II}^{+}$, and $\mathrm{III}^{-}$.

To know what happens between the speeds $F_{-}$and $F_{+}$, a more detailed analysis is required. We start by considering the solution branch $\mathrm{II}^{+}$. As we know, $] 0, F_{-}$is included in the range of speeds attained along this branch. Furthermore, the value of $\Gamma_{2}^{\prime}\left(1^{+}\right)$can switch signs depending on the parameters:

(i) If $\Gamma_{2}^{\prime}\left(1^{+}\right)<0$, then $\Gamma_{2}$ is a decreasing function in $] 1,1+1 / H[$. This is so because otherwise there would be a minimum below $F_{-}^{2}$, and consequently a collision within the range of speeds $] 0, F_{-}[$, which contradicts our findings in $\S 3.2$;

(ii) If $\Gamma_{2}^{\prime}\left(1^{+}\right)>0$, then there are exactly two critical points within the range of speeds $] F_{-}, F_{c}[$, and a single critical point within the range of speeds $] 0, F_{-}\left[{ }^{3}\right.$.

It will be proved in $\S 4$ that $\Gamma_{2}$ is monotonic in $] 1,1+1 / H\left[\right.$ if and only if $\Gamma_{3}$ is non-monotonic in ]0,1[. This means that regardless of the parameters $\rho, H$, there are within $] F_{-}, F_{c}$ [ two critical points located at the branch $\mathrm{III}^{-}$, or $\mathrm{II}^{+}$, depending whether or not $\Gamma_{2}\left(h_{2}\right)$ is monotonic in $] 1,1+1 / H[$. In addition, no critical points can be found along these two branches beyond the speed $F_{c}$.

Bearing in mind that one single non-trivial collision occurs below the speed $F=F_{+}$, we can establish that $\Gamma_{2}$ is a strictly monotonic increasing function in $] 0,1[$ (with image $] 0, F_{+}^{2}\left[\right.$ ), and $\Gamma_{3}$ is always larger than $F_{+}^{2}$ for $h_{2}>1$. In particular, if $F_{c}<F<F_{+}$, there are two critical points: the point $(1,1)$ and another point located at the branch $\mathrm{II}^{-}$.

It remains to examine the branch III $^{+}$. This branch will tend to the asymptote $h_{1}=1 / \sqrt{1-\rho}$ for large values of $h_{2} c f$. figure $5(b)$. This allows us to conclude that $\Gamma_{3}\left(h_{2}\right)$ has the same asymptotic behavior as the line equation $h_{1}=2 H h_{2}+2(1 / \sqrt{1-\rho}-1-H)$ for large values of $h_{2}$ (see figure 6 ). In particular, there can only be a single critical point on this branch beyond a certain Froude number. In addition, since $\lim _{h_{2} \rightarrow 1^{+}} \Gamma_{3}^{\prime}\left(h_{2}\right)>0$, when the function is not monotonic, local extrema come in pairs. According to $\S 3.2$, one single pair of collisions may take place at the barotropic mode (provided $H$ is small) and must correspond to a local maximum and a local minimum of $\Gamma_{3}$. Let $F_{M}^{2}, F_{m}^{2}$ be these local extrema. Then, for every value of $F$ within $] F_{m}, F_{M}$ [ there will be three critical points in this branch, as in figure $6(a)$.

This proves that regardless of the physical parameters $\rho$ and $H$, only two scenarios apply to the potential $V$. Depending on the wave speed, $V$ has two or four critical points.

\subsubsection{Classification}

In this section we classify the critical points regarding their nature. As we have shown, four critical points are obtained in the limit $F \rightarrow 0^{+}$(see figure 5 and Eq. (15)). By examining the limit value of the determinant of the Hessian matrix in (8) at each one of these points, we can conclude that for a

\footnotetext{
${ }^{3} \mathrm{~A}$ maximum of $\Gamma_{2}$ in $] 1,1+1 / H$ is strictly less than $F_{+}^{2}$, otherwise the mean value theorem would imply the existence of at least one critical point for $F=F_{+}$located at this branch, which is absurd. This extremum is also unique, by uniqueness of a non-trivial collision in $] 0, F_{+}\left[\right.$. We conclude then that such maximum is precisely $F_{c}^{2}$, and no critical points exist at the branch $\mathrm{II}^{+}$beyond the speed $F=F_{c}$.
} 


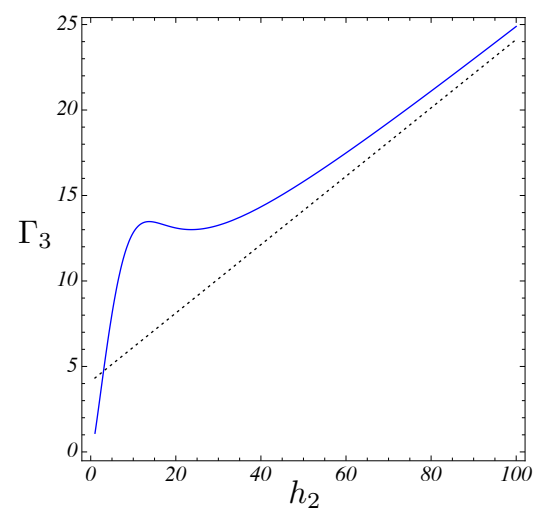

(a)

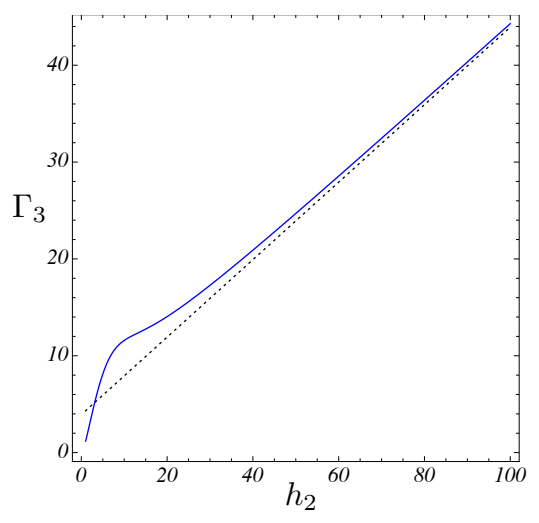

(b)

Figure 6: Monotonic and non-monotonic behavior of the function $\Gamma_{3}\left(h_{2}\right)$, with $h_{2}>1$. The graph of the function is represented by a full line, and the asymptotic behavior for large values of $h_{2}$ is represented by a dotted line. (a) $\rho=0.9, H=0.1,(b) \rho=0.9, H=0.2$.

small neighborhood of 0 , the four critical points obtained for $V$ can be classified as two saddle points (in $\mathrm{II}^{+}, \mathrm{III}^{-}$), one local minimum with coordinates $(1,1)$, and one local maximum (in $\mathrm{II}^{-}$). For given values of $\rho$ and $H$, let $F_{0}, F_{1}$ be any two values with $0<F_{0}<F_{1}$. Then, there exist $\mu, L$ such that all critical points of $V$ are contained in the square $\left.\Omega_{0}=\right] \mu, L[\times] \mu, L\left[\right.$, for all speeds within $\left[F_{0}, F_{1}\right]$. In addition, there is a value $r \ll 1$ such that if we consider for each one of the vertices of $\Omega_{0}$ a small disc $D_{i}$ with radius $r$, the closure of the set $\Omega$ defined as

$$
\Omega \equiv \operatorname{Conv}\left(\Omega_{0} \cup \bigcup_{i=1}^{4} D_{i}\right)
$$

is still contained in the interior of the first quadrant, where $\operatorname{Conv}($.$) is the convex-hull operator. \Omega$ is a bounded domain of $\mathbb{R}^{2}$ with smooth boundary $\partial \Omega$ (rounded square), where no critical points of $V$ can be found.

Let $\Phi$ be the mapping

$$
\begin{aligned}
\Phi: \partial \Omega \times[0,1] & \longrightarrow \nabla V_{\left.\right|_{F=\left(F_{1}-F_{0}\right) \theta+F_{0}}}
\end{aligned}
$$

$\Phi$ is a continuous deformation of the field $\Phi_{0} \equiv \nabla V\left(F=F_{0}\right)$ into the field $\Phi_{1} \equiv \nabla V\left(F=F_{1}\right)$. Moreover, since $\nabla V$ does not vanish in $\partial \Omega, \Phi$ is a homotopy connecting $\Phi_{0}$ and $\Phi_{1}$ on $\partial \Omega$. By Theorem 2.4.4 of [34], we conclude that $\Phi_{0}, \Phi_{1}$ have the same rotation; that is,

$$
\gamma\left(\Phi_{0} ; \partial \Omega\right)=\gamma\left(\Phi_{1} ; \partial \Omega\right) .
$$

We now consider the following Theorem by Kronecker (see Theorem 2.4.6 in [34]):

Theorem. Suppose that $\phi$ is a field defined on the closure of $\bar{\Omega}$ of a domain $\Omega$, and that $\phi$ is nondegenerate on $\partial \Omega$ and has finitely many zeros $x_{1}, \ldots, x_{k}$ in $\Omega$. Then

$$
\gamma(\phi ; \partial \Omega)=\sum_{i=1}^{k} \operatorname{ind}\left(x_{i} ; \phi\right),
$$

where $\operatorname{ind}\left(x_{i} ; \phi\right)$ is the topological index of a zero $x_{i}$ of $\phi$. 
A very simple way of computing the topological index of a zero $x_{0}$ of a field $\phi$, when $\operatorname{det} \phi^{\prime}\left(x_{0}\right) \neq 0$, is given by Theorem 4.5.2 of [35]:

$$
\operatorname{ind}\left(x_{0} ; \phi\right)=\operatorname{sign} \operatorname{det} \phi^{\prime}\left(x_{0}\right),
$$

where $\phi^{\prime}$ stands for the Jacobian matrix of $\phi$.

Let $F_{0}$ be an arbitrarily small number, and $F_{1}$ some other value greater than $F_{0}$. Consider for these two values a set $\Omega$ constructed as in (19). As we know, $V$ has precisely four critical points for $F=F_{0}$ : two saddle points and two extrema. By Kronecker's Theorem and (21), we conclude that $\gamma\left(\Phi_{0} ; \partial \Omega\right)=0$. By homotopy invariance, it follows that

$$
\gamma\left(\Phi_{1} ; \partial \Omega\right)=0 .
$$

Moreover, it can be proved numerically that the critical point at the branch $\mathrm{II}^{-}$, here denoted by $M$, is a local maximum. Putting together all the considerations made here, we conclude that:

(i) If $F_{0}<F_{1}<F_{-}$, then $(1,1)$ and $M$ are extrema of $V$ for $F=F_{1}$, and (22) holds if and only if the remaining (two) critical points are saddles;

(ii) If $F_{-}<F_{1}<F_{c}$, then $(1,1)$ is a saddle point of $V$ for $F=F_{1}, M$ is an extremum, and (22) holds if and only if the remaining (two) critical points are a saddle and an extremum;

(iii) If $F_{c}<F_{1}<F_{+}$, then $(1,1)$ is a saddle point of $V$ for $F=F_{1}$, and $M$ is an extremum;

(iv) If $F_{1}>F_{+}$and $\Gamma_{3}\left(h_{2}\right)$ is monotonic for $h_{2}>1$, then $(1,1)$ is a local maximum of $V$ for $F=F_{1}$ and the remaining critical point must be a saddle;

$(v)$ If $F_{1}>F_{+}$and $\Gamma_{3}\left(h_{2}\right)$ is non-monotonic in $] 1, \infty[$, then $(1,1)$ is a local maximum and, depending on the value of $F_{1}$, there could be three other critical points (two saddles and an extremum), or a single saddle point.

\section{Critical depth ratio and polarity of interfacial waves}

While solitary-wave solutions in a two-layer flow with a free surface are expected to be of elevation (both at the interface and top free surface) within the barotropic mode (see [25, 26]), waves in the baroclinic mode can switch polarities depending on the physical parameters (see [18, 17, 19, 20]).

A critical depth ratio separates the two different regimes. Although this critical value can be easily identified under the weakly nonlinear theory, the same cannot be said about the full nonlinear Euler equations. As a matter of fact, this value can only be inferred indirectly by examining the conjugate states [20], but intrinsic to this is the underlying assumption that front-like solutions should be the limiting form for solitary-wave solutions in the baroclinic mode. This is something far from being perfectly understood, since even in the simpler case when the top free surface is replaced by a rigid lid, a consensus has not been reached (see [36, 37, 12, 38]).

In [3], the switch of polarity of an interfacial wave has been related to a structural change of the potential $V$. Two distinct scenarios were identified for the location of critical points at speeds slightly larger than $F_{-}$. Depending on the values of physical parameters, two of critical points (a local minimum and a saddle point) could be located to the left, or to the right, of the line $h_{1}=1$ along the branch $\mathrm{II}^{+}$, or $\mathrm{III}^{-}$, as in figures $7(a),(b)$. Similarly to what was described in $\S 3$ for the particular case when $\rho=0$, homoclinic orbits are obtained, in the slow mode, by leaving the equilibrium $(1,1)$ in the directing of the minimum. Therefore, we conclude that in the former (latter) case we have a interfacial of depression (elevation).

A crucial remark is that the scenario in figure $7(b)$ (wave of elevation) occurs if and only if $\Gamma_{2}\left(h_{2}\right)$ defined in (17) is a one to one correspondence between $] 1,1+1 / H[$ and $] 0, F_{-}^{2}[$. From $\S 3.2$, this 


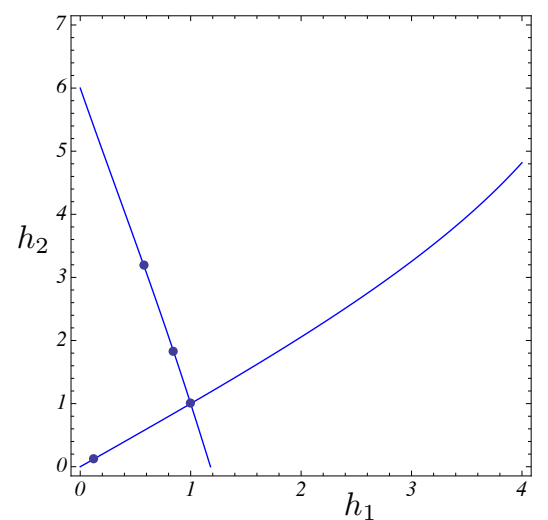

(a)

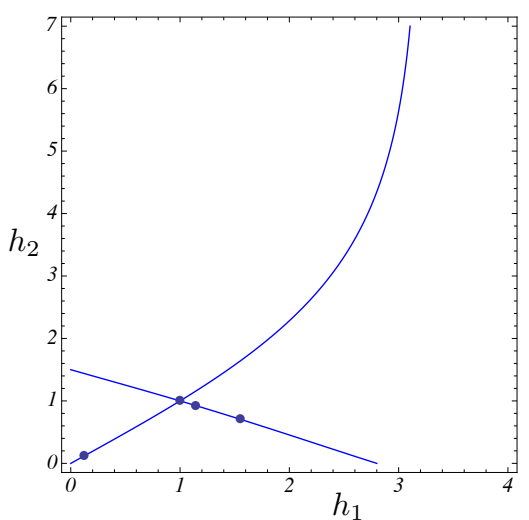

(b)

Figure 7: Two distinct scenarios for the location of critical points leading to different polarities of an interfacial wave in the baroclinic mode. $(a) \rho=0.9, H=0.2, F^{2}=0.03\left(>F_{-}^{2} \approx 0.0169\right),(b) \rho=0.9$, $H=2, F^{2}=0.074\left(>F_{-}^{2} \approx 0.0682\right)$. If solitary-wave solutions do exist for these wave speeds, $(a)$ and (b) would correspond to interfacial waves of depression and elevation, respectively. The geometrical locus defined by (11) is represented by full lines.

amounts to finding parameters for which $\Gamma_{2}^{\prime}\left(1^{+}\right)<0 . \quad \Gamma_{2}\left(h_{2}\right)$ is differentiable in $] 1,1+1 / H$ [, with derivative

$$
\Gamma_{2}^{\prime}\left(h_{2}\right)=2 h_{2}\left[\frac{2 \Lambda_{2}\left(h_{2}\right)+h_{2}\left(1-h_{2}^{2}\right) \Lambda_{2}^{\prime}\left(h_{2}\right)}{\left(1-h_{2}^{2}\right)^{2}}\right] .
$$

Hence,

$$
\lim _{h_{2} \rightarrow 1^{+}} \Gamma_{2}^{\prime}\left(h_{2}\right)=-\frac{1}{2}\left[-3 F_{-}^{2}-3 H(\rho-1)\left(\frac{H-1+\sqrt{(H-1)^{2}+4 \rho H}}{2 \sqrt{(H-1)^{2}+4 \rho H}}\right)\right] .
$$

The condition $\Gamma_{2}^{\prime}\left(1^{+}\right)<0$ is then equivalent to

$$
2 F_{+}^{4}-[H(1-\rho)-3(H+1)] F_{+}^{2}+(H+1)^{2}-H(1-\rho)>0,
$$

which holds precisely when

$$
H^{3}-\left(3-3 \rho-\rho^{2}\right) H^{2}+(3-4 \rho) H-1>0 .
$$

When the opposite inequality holds, it can be proved that $\Gamma_{3}^{\prime}\left(1^{-}\right)>0$, in which case $\Gamma_{3}\left(h_{2}\right)$ is strictly monotonic increasing in ]0,1[ and $\mathrm{III}^{-}$has no critical points beyond $F=F_{c}$ (cf. figure $7(a)$ ). This establishes that: $\Gamma_{2}$ is monotonic in $] 1,1+1 / H$ [ if and only if $\Gamma_{3}$ is non-monotonic in ]0,1[; the critical depth ratio ruling the polarity of the interfacial waves is given by the single real root of the cubic equation

$$
H^{3}-\left(3-3 \rho-\rho^{2}\right) H^{2}+(3-4 \rho) H-1=0 .
$$

This is in perfect agreement with the value found in earlier models under the weakly nonlinear hypothesis (see e.g. Kakutani \& Yamasaki [19]). Furthermore, since no critical points can be found in $\mathrm{II}^{+}$or $\mathrm{III}^{-}$beyond the speed $F=F_{c}$, solitary waves cannot exist within the range $] F_{c}, F_{+}[$.

\section{Conjugate states: exact vs approximate theory}

We consider the Euler equations and assume the existence of a front wave moving from left to right at constant speed $\mathrm{c}$ into a two-layer stratified fluid at rest at $\xi=-\infty$. Thus, in a frame moving with 
such a right-going front of speed $c$, the velocity at $\xi=-\infty$ is $c$ in both fluids, i.e., $u_{1}=u_{2}=c$. On the other hand, the velocity at $\xi=+\infty$ can be expected to be different, say $u_{1}^{\mathrm{d}}\left(u_{2}^{\mathrm{d}}\right)$, in an lower (upper) fluid layer whose thickness is $h_{1}^{\mathrm{d}}\left(h_{2}^{\mathrm{d}}\right)$. For front-like solutions to exist, there should exist $c, u_{i}^{\mathrm{d}}$ and $h_{i}^{\mathrm{d}}$, for given $\rho_{i}$ and $H_{i}, i=1,2$, such that all three basic physical conservation laws of mass, momentum and energy hold. This problem has been addressed by Dias \& Il'ichev [20] and is a particular case of the detailed analysis of conjugate flows by Lamb [27] in a three-layer configuration. We stress that only the upstream and downstream states are considered, and no attempt is made to describe the shape of such solutions.

To solve the problem, the authors in [20] arrive to a set of three algebraic equations (A.15)-(A.17) for three unknowns. It is straightforward to check that (A.15), (A.16) are equivalent to $\frac{\partial V}{\partial h_{1}}\left(h_{1}^{\mathrm{d}}, h_{2}^{\mathrm{d}}\right)=0$ and $\frac{\partial V}{\partial h_{2}}\left(h_{1}^{\mathrm{d}}, h_{2}^{\mathrm{d}}\right)=0$, respectively. Similarly, it can be checked that (A.17) is equivalent to

$$
V\left(h_{1}^{\mathrm{d}}, h_{2}^{\mathrm{d}}\right)+h_{1}^{\mathrm{d}} \frac{\partial V}{\partial h_{1}}\left(h_{1}^{\mathrm{d}}, h_{2}^{\mathrm{d}}\right)+h_{2}^{\mathrm{d}} \frac{\partial V}{\partial h_{2}}\left(h_{1}^{\mathrm{d}}, h_{2}^{\mathrm{d}}\right)=0 .
$$

As a consequence, the conjugate states for Euler equations are precisely the same as those for the strongly nonlinear model, and the corresponding traveling-wave solutions, if they exist, correspond precisely to heteroclinic connections between the critical points $(1,1)$ and $\left(h_{1}^{\mathrm{d}}, h_{2}^{\mathrm{d}}\right)$, being the latter a non-trivial solution of

$$
V\left(h_{1}, h_{2}\right)=0, \quad \nabla V\left(h_{1}, h_{2}\right)=0
$$

Making use of the notation

$$
H_{1}^{\star}=1 /(1+H), \quad H_{2}^{\star}=H /(1+H), \quad H_{1}^{\star^{\prime}}=h_{1}^{\mathrm{d}} /(1+H), \quad H_{2}^{\star \prime}=H h_{2}^{\mathrm{d}} /(1+H), \quad\left(F^{\star}\right)^{2}=F^{2} /(1+H),
$$

the authors in [20] have used (A.15) to express $H_{2}^{\star \prime}$ as a function of $H_{1}^{\star \prime}$ and $F^{\star}$, and plugged this into the remaining equations (A.16)-(A.17). These consist of polynomial equations on $H_{1}^{\star \prime}$ (of degree 5 and 8 , respectively), required to have a common root. For this to happen, the resultant of both polynomials must vanish, that is:

$$
\left[\left(F^{\star}\right)^{4}-\left(F^{\star}\right)^{2}+(1-\rho) H_{1}^{\star}\left(1-H_{1}^{\star}\right)\right] Q_{8}\left(H_{1}^{\star}\right)=0 .
$$

The first term on the left-hand side is just the left-hand side of Eq. (2) and corresponds to trivial conjugate states. The roots of the eight-degree polynomial $Q_{8}$ (defined in the Appendix B) yield the non-trivial solutions of (24), which for fixed values of $\rho$ can be represented on the $\left(H_{1}^{\star}, F^{\star}\right)$-plane as curves (hereafter referred to as "front" curves) as in figures 2, 3.

Dias \& Il'ichev [20] claimed that it can be shown numerically that for all values of $\rho$ between 0 and 1 and for all values of $H_{1}^{\star}$ between 0 and 1 , there is always a unique solution for $F^{\star}$. As it can be seen from figure 3 , the statement is not entirely accurate although, for the same reasons evoked earlier (see $\S 3.2$ ), it may be that other solutions should be discarded for lack of physical meaning. Nonetheless, we emphasize that at least one of these solutions should be retained, as front-like solutions are expected to exist in the baroclinic mode as a consequence of the well-known broadening phenomenon. In this case it is desirable to have at our disposal a good estimate of these speed values without having to go through these messy expressions. Since both in oceanic conditions and laboratory experiments the density ratio is typically between 0.8 and 1 , we can let $\rho=1-\varepsilon(\varepsilon \ll 1)$ and use asymptotics to make predictions.

Remark that the coefficients of $Q_{8}$ are polynomials on the variable $F^{\star 2}$ (see Appendix B). That being so, we can look alternatively at the equation $Q_{8}\left(H_{1}^{\star}\right)=0$ as a polynomial equation $Q_{7}\left(F^{\star 2}\right)=0$ of degree 7 for $F^{\star 2}$. We convert everything back to our notation, and seek for roots of $\widetilde{Q}_{7}\left(F^{2}\right)$ with the form

$$
F_{\text {front }}^{2}=C_{0}+\varepsilon C_{1}+\varepsilon^{2} C_{2}+O\left(\varepsilon^{3}\right) .
$$

At leading order, we have

$$
(1+H) C_{0}\left(C_{0}-4-4 H\right)^{3}\left[C_{0}^{3}-3(1+H) C_{0}^{2}+3\left(1-7 H+H^{2}\right) C_{0}-(1+H)^{3}\right]=0,
$$


whose real roots, besides zero, are larger than $1+H\left(>F_{+}^{2}\right)$. The asymptotics reveal that for small density contrasts there can only exist in the baroclinic mode a single solution $F=F_{\text {front }}$ of $\widetilde{Q}_{7}\left(F^{2}\right)=0$. Moreover, $F_{\text {front }}^{2}=O(\varepsilon)$, since the leading order is zero. First and second order corrections can be easily determined, leading to

$$
F_{\text {front }}^{2}=\varepsilon\left(\frac{1+H}{4}\right)+\varepsilon^{2}\left(\frac{7+10 H-H^{2}}{64(1+H)}\right)+O\left(\varepsilon^{3}\right) .
$$

\section{Discussion}

This work is devoted to a better understanding of the mathematical properties of the model for large amplitude internal waves in a two-layer system with a trop free surface proposed in $[1,2]$. Its solitary-wave solutions were studied in [3], but important open questions remained to be addressed. To overcome some of these problems, a rigorous critical point analysis is employed in this study. The geometrical locus, number, and nature of the critical points of the Hamiltonian system governing the solitary-wave solutions of (1) are found here. In particular, it is shown that regardless of density and depth ratios there exist two or four critical points, depending on the wave speed. Knowing their location is key to the understanding how a structural change of the potential occurs for different physical parameters $\rho$ and $H$, which in fact determines the polarity of an interfacial wave in the baroclinic mode. The critical depth ratio distinguishing the two regimes (of elevation or depression of the interface) is derived explicitly from the model and it is shown to be in perfect agreement with the results in the literature for weakly nonlinear models (see $\S 4$ ).

By examining when a collision between critical points occur, we are able to show that solitary-wave solutions in the slow mode $\left(F_{-}<F<F_{+}\right)$can never exist for the whole range of speeds, being $F=F_{c}$ an upper bound for their speed (see $\S 3.2$ ). If the limiting form of solitary-wave solutions is a front-like solution connecting two conjugate states (found in $\S 5$ to be the same as those predicted by the Euler equations), then such upper bound is not sharp and solutions cease to exist at the speed $F=F_{\text {front }}<F_{\mathrm{c}}$, as conjectured by Dias \& Il'ichev (2001) for the fully nonlinear Euler equations (see shaded region in figures 2,3 ). If not, it would be interesting to investigate when the realm of existence of solutions can be extended up to the collision curve, and what these look like within the range $] F_{\text {front }}, F_{c}[$.

Some surprising results are also revealed in this manuscript. One is the presence (for certain parameters) of four critical points in the barotropic mode, which had not been detected previously. The other relates to the existence of conjugate states in barotropic mode. Dias \& Il'ichev [20] have stated that these can never exist in the barotropic mode, which is certainly true in the case when $\Gamma_{3}\left(h_{2}\right)$ in (17) is monotonic in $] 1, \infty\left[\right.$, in view of the fact that the two critical points in $\mathrm{III}^{+}$have different energy levels. Heteroclinic connections between two equilibria can also be ruled out even when monotonicity is violated, provided $\rho$ is large enough $c f$. figure $3(c)$, because the "front" curve in the barotropic mode is always below the "collision" curves. However, in a situation where a "front" curve is between two "collision" curves (see dark shaded regions in figures $3(a),(b)$ ), there is room for questioning the validity of the claim made by the authors. A more careful investigation is needed to draw any conclusion on this issue.

\section{Acknowledgments}

The author thanks W. Choi for helpful discussions and valuable suggestions, and anonymous referees for important remarks that improved the paper. The author would also like to thank Instituto Nacional de Matemática Pura e Aplicada (IMPA) for the use of MathematicA. The author acknowledges the support of MACSI, the Mathematics Applications Consortium for Science and Industry (www.macsi.ul.ie) funded by the Science Foundation Ireland Investigator Award 12/IA/1683, and the Irish Research Council through the "New Foundations' Scheme 2014". 


\section{Appendix A. Parameterizing the geometrical locus of critical points}

Define $A_{1}, A_{2}, A_{3}$ such that $P\left(h_{1}, h_{2}\right)=h_{1}^{3}+A_{1} h_{1}^{2}+A_{2} h_{1}+A_{3}, c f$. Eq. (11). Introduce the quantities

$$
A_{1} \equiv 3 a, \quad A_{2} \equiv 3 b, \quad \alpha \equiv a^{2}-b, \quad \beta \equiv 2 a^{3}-3 a b+A_{3},
$$

and let $h_{1}=x-a$ to rewrite $(11)$ as

$$
x^{3}-3 \alpha x+\beta=0 .
$$

Then, by using the discriminant, the solutions of (A.1) are all real provided that $|\beta| \leqslant 2 \alpha^{3 / 2}$, and are complex (two of them) otherwise. The values for $\alpha$ and $\beta$ are in our case given by:

$$
\begin{aligned}
& \alpha=\frac{1}{9}\left\{3 h_{2}^{2}+\left[1+H\left(h_{2}-1\right)\left(h_{2}^{2}(\rho-1)-\rho\right)\right]^{2}\right\}=a^{2}+\frac{1}{3} h_{2}^{2}>0 \\
& \beta=h_{2}^{2}\left[1+H\left(1-h_{2}\right)\right]-\frac{1}{3} h_{2}^{2}\left[1+H\left(h_{2}-1\right)\left[h_{2}^{2}(\rho-1)-\rho\right]-\frac{2}{27}\left[1+H\left(h_{2}-1\right)\left[h_{2}^{2}(\rho-1)-\rho\right]\right]^{3},\right.
\end{aligned}
$$

and based on figure 1, where two solution branches can be seen, it is certainly the case when all roots are real. We use trigonometric functions ( $c f$. Miura [31]) to write the roots of (11) as:

$$
\begin{aligned}
& h_{1}=\lambda_{1}\left(h_{2}\right) \equiv-2 \sqrt{\alpha} \sin (\pi / 3+\phi)-a, \\
& h_{1}=\lambda_{2}\left(h_{2}\right) \equiv 2 \sqrt{\alpha} \sin \phi-a, \\
& h_{1}=\lambda_{3}\left(h_{2}\right) \equiv 2 \sqrt{\alpha} \sin (\pi / 3-\phi)-a,
\end{aligned}
$$

with $\phi=\frac{1}{3} \arcsin \left(\frac{\beta}{2 \alpha^{3 / 2}}\right)$. Since $|\beta| / 2 \alpha^{3 / 2} \leqslant 1$, we have $-\pi / 6 \leqslant \phi \leqslant \pi / 6$, and hence $\lambda_{1}\left(h_{2}\right) \leqslant$ $-\sqrt{\alpha}-a$. This fact can be used to prove that $\lambda_{1}\left(h_{2}\right)<0$. If $a>0$, then the claim is trivial. On the other hand, if $a<0$, because $\alpha-a^{2}=h_{2}^{2} / 3 \geqslant 0$, we have $\sqrt{\alpha} \geqslant|a|$, i.e., $\sqrt{\alpha} \geqslant-a$. In either case, we may conclude that $\lambda_{1}\left(h_{2}\right)<0$. Therefore, the solution branches relevant to our study are parametrized by $h_{1}=\lambda_{2}\left(h_{2}\right)$ and $h_{1}=\lambda_{3}\left(h_{2}\right)$, cf. figure 5 .

\section{Appendix B. Conjugate states. Coefficient list for the polyno- mial $Q_{8}$ in $(25)$}

The polynomial equation $Q_{8}\left(H_{1}^{\star}\right)=0$ in (25) implicitly defines the wave speeds at which non-trivial conjugate states exist. This polynomial, originally denoted by $P_{2}$ in Eq. (A.20) of [20], is an eightdegree polynomial on $H_{1}^{\star}$, i.e., $Q_{8}=\sum_{k=0}^{8} a_{k} H_{1}^{\star 8-k}$. Only the higher-order coefficients $a_{0}, a_{1}$ were listed in [20]. Given the importance of knowing these speed values, we include here the remaining coefficients:

$$
\begin{gathered}
a_{0}=16(\rho-1)^{5}, \\
a_{1}=96(\rho-1)^{4}(2 \rho+1) F^{\star 2}, \\
a_{2}=8(\rho-1)^{2}\left(F^{\star 4}(\rho(\rho(76 \rho-245)-17)-30)+8 F^{\star 2} \rho((5-7 \rho) \rho+2)-8(\rho-1)^{2} \rho\right), \\
a_{3}=8 F^{\star 2}(\rho-1)^{2}\left(F^{\star 4}(\rho(\rho(24 \rho-353)-8)+40)-8 F^{\star 2} \rho(\rho(43 \rho-80)+10)+4 \rho\left(-2 \rho^{2}+\rho+1\right)\right), \\
a_{4}=(\rho-1)\left(F^{\star 8}(\rho(4 \rho(2 \rho(2 \rho-91)+243)-527)+240)-4 F^{\star 6} \rho\left(\rho\left(208 \rho^{2}-2682 \rho+1641\right)+293\right)+\right. \\
\left.+8 F^{\star 4} \rho(\rho(\rho(628 \rho-1101)+942)-37)+64 F^{\star 2}(\rho-1) \rho^{2}(13 \rho+6)+96(\rho-1)^{2} \rho^{2}\right),
\end{gathered}
$$




$$
\begin{gathered}
a_{5}=-F^{\star 2}(\rho-1)\left(F^{\star 8}(\rho(8 \rho(7 \rho+12)-221)+96)+4 F^{\star 6} \rho(4 \rho(\rho(4 \rho-151)+146)-239)-\right. \\
\left.-8 F^{\star 4} \rho(2 \rho(\rho(88 \rho-903)+261)+55)+128 F^{\star 2} \rho^{2}(\rho(37 \rho-40)+30)+224(\rho-1) \rho^{2}(2 \rho+1)\right), \\
a_{6}=F^{\star 12}(1-\rho)(\rho(12 \rho+5)-16)+F^{\star 10} \rho(\rho(12 \rho(14 \rho+9)-565)+316)+ \\
+4 F^{\star 8} \rho(\rho(6 \rho(4(\rho-31) \rho+183)-505)+46)+8 F^{\star 6} \rho^{2}(\rho(2(643-72 \rho) \rho-1061)+81)+ \\
\quad+8 F^{\star 4} \rho^{2}(\rho(\rho(308 \rho-647)+183)-60)-64 F^{\star 2} \rho^{3}\left(5 \rho^{2}+\rho-6\right)-64(\rho-1)^{2} \rho^{3}, \\
a_{7}=\rho F^{\star 2}\left(F^{\star 12}(-(\rho-1))+4 F^{\star 10}(\rho-1)(6 \rho+5)-F^{\star 8}(\rho(24 \rho(7 \rho+4)-245)+8)+\right. \\
\quad+4 F^{\star 6} \rho(57-4 \rho(\rho(4 \rho-93)+83))+8 F^{\star 4} \rho(\rho(\rho(56 \rho-401)+174)+9)+ \\
\left.\quad+64 F^{\star 2} \rho^{2}((32-11 \rho) \rho+6)+160(\rho-1) \rho^{2}(2 \rho+1)\right), \\
a_{8}=\rho^{2}\left(F^{\star 2}-1\right)^{3}\left(F^{\star 8}-12 \rho F^{\star 6}+8 F^{\star 4} \rho(7 \rho-1)+16 F^{\star 2}(\rho-5) \rho^{2}-16(\rho-1) \rho^{2}\right) .
\end{gathered}
$$

\section{References}

[1] W. Choi and R. Camassa, Weakly nonlinear internal waves in a two-fluid system, J. Fluid Mech. 313:83103 (1996).

[2] R. Barros, S. L. Gavrilyuk, and V. M Teshukov, Dispersive nonlinear waves in two-layer flows with free surface. I. Model derivation and general properties, Stud. Appl. Math. 119:191-211 (2007).

[3] R. Barros and S. L. Gavrilyuk, Dispersive nonlinear waves in two-layer flows with free surface. II. Large amplitude solitary waves embedded into the continuous spectrum, Stud. Appl. Math. 119:213-251 (2007).

[4] R. Grimshaw, Internal solitary waves, Environmental stratified flows. Springer US, 1-27, 2002.

[5] K. R. Helfrich and W. K. Melville, Long nonlinear internal waves, Annu. Rev. Fluid Mech. 38:395-425 (2006).

[6] M. Mryata, Long internal waves of large amplitude, in: H. Horikawa, H. Maruo, (Eds.), Proc. of the IUTAM Symp. on Nonlinear Water Waves, pp. 399-406, 1988.

[7] W. Choi and R. Camassa, Fully nonlinear internal waves in a two-fluid system, J. Fluid Mech. 396:1-36 (1999).

[8] J. L. Bona, D. Lannes, and J.-C. SAut, Asymptotic models for internal waves, J. Math. Pures Appl. 89:538-566 (2008).

[9] W. Craig, P. Guyenne, and H. Kalisch, A new model for large amplitude long internal waves, Comptes Rendus Mecanique 332:525-530 (2004).

[10] W. Choi, R. Barros, and T-C. Jo, A regularized model for strongly nonlinear internal solitary waves, J. Fluid Mech. 629:73-85 (2009).

[11] R. Camassa, W. Choi, H. Michallet, P. Rusas, and J. K. Sveen, On the realm of validity of strongly nonlinear asymptotic approximations for internal waves, J. Fluid Mech. 549:1-23 (2006).

[12] W. A. B. Evans and M. J. Ford, An integral equation approach to internal (2-layer) solitary waves, Phys. Fluids 8:2032-2047 (1996).

[13] R. Liska, L. Margolin, and B. Wendroff, Nonhydrostatic two-layer models of incompressible flow, Computers Math. Applic. 29:25-37 (1995). 
[14] W. Craig, P. Guyenne, and H. Kalisch, Hamiltonian long-wave expansions for free surfaces and interfaces, Comm. Pure Appl. Math. 58:1587-1641 (2005).

[15] J. R. Percival, C. J. Cotter, and D. D. Holm, A Euler-Poincaré framework for the multilayer GreenNagdhi equations, J. Phys. A: Mathematical and Theoretical 41:344018 (2008).

[16] V. Duchêne, Asymptotic shallow water models for internal waves in a two-fluid system with a free surface, SIAM Journal on Mathematical Analysis 42:2229-2260 (2010).

[17] L. R. WALker, Interfacial solitary waves in a two-fluid medium, Phys. Fluids 16:1796-1804 (1973).

[18] A. S. Peters and J. J. Stoker, Solitary waves in liquids having non-constant density, Comm. Pure Appl. Math. 13:115-164 (1960).

[19] T. Kakutani and N. Yamasaki, Solitary waves on a two-layer fluid, J. Phys. Soc. Jpn. 45:674-679 (1978).

[20] F. DiAs and A. IL'ICHEV, Interfacial waves with free-surface boundary conditions: an approach via a model equation, Physica D 150:278-300 (2001).

[21] H. Michallet and F. Dias, Numerical study of generalized interfacial solitary waves, Phys. Fluids 11:1502-1511 (1999).

[22] F. Dias and G. Iooss, Water waves as a spatial dynamical system, in Handbook of Mathematical Fluid Dynamics, Chapter 10, pp. 443-499 (S. Friedlander and D. Serre, Eds.) Elsevier, North Holland, 2003.

[23] R. Grimshaw, Models for long-wave instability due to a resonance between two waves, in Trends in Applications of Mathematics to Mechanics (G. Iooss, O. Guès, and A. Nouri, Eds.), Chapman \& Hall/CRC, 2000 .

[24] C. Fochesato, F. Dias, and R. Grimshaw, Generalized solitary waves and fronts in coupled Korteweg-de Vries systems, Physica D 210:96-117 (2005).

[25] J. N. Moni and A. C. KInG, Guided and unguided interfacial solitary waves, The Quarterly Journal of Mechanics and Applied Mathematics 48:21-38 (1995).

[26] H. C. Woolfenden and E. I. PArau, Numerical computation of solitary waves in a two-layer fluid, J. Fluid Mech. 688: 528 (2011).

[27] K. G. Lamb, Conjugate flows for a three-layer fluid, Phys. Fluids 12:2169-2185 (2000).

[28] C. H. Su and C. S. Gardner, Korteweg-de Vries Equation and Generalizations. III. Derivation of the Korteweg-de Vries Equation and Burgers Equation, J. Math. Phys. 10:536-539 (1969).

[29] A. E. GREen and P. M. NAGHDI, A derivation of equations for wave propagation in water of variable depth, J. Fluid Mech. 78:237-246 (1976).

[30] R. Osborne and T. L. Burch, Internal solitons in the Andaman Sea, Science 4443:451-460 (1980).

[31] R. M. Miura, Explicit roots of the cubic polynomial and applications, Appl. Math. Notes 4:22-40 (1980).

[32] V. V. Prasolov, Polynomials, Algorithms and Computation in Mathematics, Springer, 2004.

[33] E. J. Primrose, Plane Algebraic Curves, Macmillan, London, UK, 1955.

[34] S. V. Emelyanov, S. K. Korovin, N. A. Bobylev, and A. V. Bulatov, Homotopy of Extremal Problems: Theory and Applications, De Gruyter, 2007.

[35] N. A. Bobylev, S. V. Emelyanov, and S. K. Korovin, Geometrical Methods in Variational Problems, Vol. 485, Springer, 1999.

[36] R. Grimshaw and D. Pullin, Extreme interfacial waves, Phys. Fluids 29:2802 (1986).

[37] R. Turner and J. VAnden-Broeck, Broadening of interfacial solitary waves, Phys. Fluids 31:286 (1988).

[38] F. Dias and J-M. Vanden-Broeck, On internal fronts, J. Fluid Mech. 479:145-154 (2003). 